\title{
Recent Administration of lodinated Contrast Renders Core Infarct Estimation Inaccurate Using RAPID Software
}

\author{
(D) A.Z. Copelan, (DE.R. Smith, (D) G.T. Drocton, (D).H. Narsinh, (DD. Murph, (D)R.S. Khangura, (D) Z.J. Hartley, (D)A.A. Abla, \\ (D)W.P. Dillon, (D) C.F. Dowd, (D) R.T. Higashida, (D)V.V. Halbach, (D) S.W. Hetts, DD.L. Cooke, (D) K. Keenan, (D). Nelson, (DD. Mccoy, \\ (D) M. Ciano, and (D) M.R. Amans

\begin{abstract}
BACKGROUND AND PURPOSE: Automated CTP software is increasingly used for extended window emergent large-vessel occlusion to quantify core infarct. We aimed to assess whether RAPID software underestimates core infarct in patients with an extended window recently receiving IV iodinated contrast.
\end{abstract}

MATERIALS AND METHODS: We reviewed a prospective, single-center data base of 271 consecutive patients who underwent CTA \pm CTP for acute ischemic stroke from May 2018 through January 2019. Patients with emergent large-vessel occlusion confirmed by CTA in the extended window ( $>6$ hours since last known well) and CTP with RAPID postprocessing were included. Two blinded raters independently assessed CT ASPECTS on NCCT performed at the time of CTP. RAPID software used relative cerebral blood flow of $<30 \%$ as a surrogate for irreversible core infarct. Patients were dichotomized on the basis of receiving recent IV iodinated contrast $(<8$ hours before (TP) for a separate imaging study.

RESULTS: The recent IV contrast and contrast-naïve cohorts comprised 23 and 15 patients, respectively. Multivariate linear regression analysis demonstrated that recent IV contrast administration was independently associated with a decrease in the RAPID core infarct estimate (proportional increase $=0.34 ; 95 \% \mathrm{Cl}, 0.12-0.96 ; P=.04$ ).

CONCLUSIONS: Patients who received IV iodinated contrast in proximity ( $<8$ hours) to CTA/CTP as part of a separate imaging study had a much higher likelihood of core infarct underestimation with RAPID compared with contrast-naïve patients. Over-reliance on RAPID postprocessing for treatment disposition of patients with extended window emergent large-vessel occlusion should be avoided, particularly with recent IV contrast administration.

ABBREVIATIONS: ELVO = emergent large-vessel occlusion; LKW = last known well; MT = mechanical thrombectomy; PI = proportional increases; $\mathrm{rCBF}=$ relative cerebral blood flow

$\mathbf{Q}$ uantifying core infarction versus viable ischemic penumbra is at the crux of patient selection for mechanical thrombectomy (MT) in the setting of anterior circulation emergent largevessel occlusion (ELVO). While patients with large infarcts tend to demonstrate worse clinical outcomes following reperfusion, successful recanalization of sizable ischemic penumbra, indicative of salvageable tissue, may result in drastic clinical improvement. ${ }^{1}$

Received January 24, 2020; accepted after revision August 1.

From the Departments of Diagnostic and Interventional Neuroradiology (A.Z.C., E.R.S., G.T.D., K.H.N., D.M., R.S.K., Z.J.H., A.A.A., W.P.D., C.F.D., R.T.H., V.V.H., S.W.H., D.L.C., J.N., D.M., M.C., M.R.A.), and Neurosurgery (A.A.A.), University of California, San Francisco, San Francisco, California; Department of Radiology (E.R.S.), Medical College of Wisconsin, Milwaukee, Wisconsin; and Department of Neurology (K.K.), University of California Davis, Sacramento, California.

Paper previously presented at: Annual Meeting of the Society of Neurointerventional Surgery, July 21-26, 2019; Miami, Florida.

Research reported in this publication was supported by the National Institute on Deafness and Other Communication Disorders of the National Institutes of Health under award No. R21DC016087-01A1 and the National Heart, Lung, and Blood Institute of the National Institutes of Health under award number R56HL149124-01.
Segregation of core infarction from ischemic penumbra is particularly relevant for extended window ELVOs ( $>6$ hours since last known well [LKW])., ${ }^{2,3}$

The semiquantitative ASPECTS system is highly predictive of clinical outcome with ELVO but demonstrates high inter- and intrareader variability. ${ }^{4,5}$ Additionally, ASPECTS regions are volumetrically weighted unequally; consequently, patients with the same ASPECTS may have different core infarct volumes depending on the regions involved. The automated quantitative RApid processing of PerfusIon and Diffusion (RAPID; iSchemaView) CTP platform offers standardized and numeric estimation of core infarct and ischemic penumbra, lessening reliance on neuroradiologic ASPECTS interpretation. RAPID estimates a variety of

Please address correspondence to Matthew R. Amans, MD, MSc, Department of Diagnostic and Interventional Neuroradiology, 505 Parnassus Ave, San Francisco, CA 94143; e-mail: Matthew.amans@ucsf.edu; @UCSFimaging

- Indicates open access to non-subscribers at www.ajnr.org

http://dx.doi.org/10.3174/ajnr.A6908 
perfusion parameters indicative of cerebral hemodynamics at the moment of scanning. Accordingly, RAPID may predict tissue fate in the hyperacute setting ( $<1$ hour since LKW). NCCT, however, is dependent on parenchymal hypoattenuation, which becomes apparent at least several hours from symptom onset.

RAPID software has been validated in multiple clinical trials, notably in DAWN (DWI or CTP Assessment with Clinical Mismatch in the Triage of Wake-Up and Late Presenting Strokes Undergoing Neurointervention with Trevo) and DEFUSE-3 (Endovascular Therapy Following Imaging Evaluation for Ischemic Stroke 3), both using RAPID for patient selection for MT in extended window ELVOs. Patients allocated to MT versus best medical therapy alone in the DAWN and DEFUSE- 3 trials demonstrated markedly better clinical outcomes with unprecedented numbers needed to treat (NNT) of 2.8 and 4, respectively, to achieve functional independence at 90 days. ${ }^{2,3}$

Through more ubiquitous RAPID use, we encountered a recurrent phenomenon in which transfer patients with extended window ELVO demonstrated MCA territory hypoattenuation on NCCT but with disproportionately small and, in some instances, zero RAPID estimated core infarct. Essentially, all ELVO transfers to our institution are recent recipients of IV iodinated contrast. Given this imaging incongruity and our ongoing need to optimize patient selection for MT, we aimed to assess whether RAPID software underestimated core infarct volume in patients who received recent ( $<8$ hours) IV contrast for a separate imaging study, most commonly CTA \pm CTP at an outside hospital before transfer.

\section{MATERIALS AND METHODS}

These study data contain sensitive personal information and cannot be made publicly available according to local data-protection regulations.

\section{Patient Selection}

We performed a retrospective review of a prospectively maintained acute ischemic stroke data base from a tertiary care academic institution (University of California, San Francisco). We reviewed 271 consecutive patients presenting with acute ischemic stroke symptoms who underwent CTA \pm CTP at our institution from May 2018 to January 2019. Inclusion criteria comprised extended window presentation; baseline NCCT, CTA confirming ELVO (ICA terminus, MCA M1 and M2 segments); and technically adequate CTP imaging (absence of excessive patient motion, poor cardiac output, or incorrect selection of arterial input function or venous output function on review of arterial input function/venous output function time plots), with RAPID postprocessing and availability of demographic and clinical data. Three transfer patients received IV tPA at an outside institution; however, all 3 had persistent ELVO on repeat imaging at our institution. An ASPECTS of 10 was excluded because by definition, these patients could not harbor a falsely low RAPID estimated core infarct volume. Thirty-eight patients met the inclusion criteria, with most exclusions due to absence of ELVO on CTA, imaging performed within 6 hours since LKW, and an ASPECTS of 10. This study was approved by the local institutional review board, with the board waiving the need for patient consent.

Two neurointerventional radiologists (A.Z.C., G.T.D.) with subspecialty neuroradiology fellowship training, blinded to clinical data as well as additional imaging and therapeutic interventions at the time of review, independently assigned an ASPECTS (0-10) to NCCTs and collateral scores to corresponding CTAs $(1=$ excellent, $2=$ intermediate, $3=$ poor $){ }^{6}$ CTP imaging was processed with fully automated, commercially available RAPID software, Version 4.5.0., to define tissue state, with ischemic core defined as relative $\mathrm{CBF}(\mathrm{rCBF})$ of $<30 \%$ of the corresponding contralateral territory (ie, CBF is comparatively reduced $>70 \%)^{7}$

Demographic and clinical data were collected including, age, sex, hours since LKW, serum creatinine level, and whether MT was performed. In addition, CT ASPECTS, CTA collaterals grade, and core infarct volume (milliliters) by RAPID were collected for each patient. We also obtained the time from CTP to reperfusion.

\section{Imaging Protocols}

Imaging Acquisition. Patients underwent an institutional stroke imaging protocol, including NCCT, CTA, and CTP performed on a 40-mm, 64-detector row clinical system (LightSpeed VCT; GE Healthcare). Helical NCCT (120 kV; 300-355 auto-mA) was performed from the foramen magnum to the vertex at a $3.75-\mathrm{mm}$ section thickness. Helical CTA (120 kV; 200-700 auto-mA) was performed from the mid-cardiac level to the vertex (section thickness/interval, $0.625 / 0.5 \mathrm{~mm}$ ) after intravenous administration of $70 \mathrm{~mL}$ of iodinated contrast, power-injected at $5 \mathrm{~mL} / \mathrm{s}$ through an 18- or 20-ga antecubital IV access and followed by a 25 -mL saline flush. After 1-2 minutes, 2 contiguous CTP slabs were obtained for $8-\mathrm{cm}$ combined supratentorial coverage, obtained at eight 5$\mathrm{mm}$ sections per slab. For each of the 2 slabs, obtained approximately 90 seconds apart, cine mode acquisition $(80 \mathrm{kV} ; 100 \mathrm{~mA})$ permitting high-temporal resolution (1-second sampling interval) dynamic bolus passage imaging was obtained after the administration of $40-\mathrm{mL}$ of iodinated contrast, power-injected at $5 \mathrm{~mL} / \mathrm{s}$. Contrast administration was followed by a $25-\mathrm{mL}$ saline flush at the same rate. Subsequently, delayed contrast-enhanced CT $(120 \mathrm{kV}$; 300-355 auto-mA) was performed from the foramen magnum to the vertex at $3.75-\mathrm{mm}$ thickness. In total, $150 \mathrm{~mL}$ of iodinated contrast was administered.

Imaging Processing. Images were processed with fully automated, commercially available CTP software (RAPID, Version 4.5.0.) to identify and distinguish potentially salvageable ischemic penumbra (delay of the maximum of the tissue residue function longer than 6 seconds, time-to-maximum of $>6$ seconds) from irreversibly infarcted core ( $\mathrm{rCBF}$ of $<30 \%$ compared with normal tissue). We also manually obtained final infarct volumes in patients treated with embolectomy with TICI $2 \mathrm{~b}, 2 \mathrm{c}$, or 3 results using OsiriX Imaging Software 11.0 (http://www.osirix-viewer.com), blinded to each patient's cohort (contrast-naïve versus received prior contrast).

Statistical Analysis. Patients were dichotomized into 2 cohorts: recent ( $<8$ hours) recipients of IV iodinated contrast before CTP at our institution versus contrast-naïve patients. Power analysis was performed on both cohorts, with a 1-to-1 enrollment ratio and binomial end points. We assumed 5\% overestimation in the noncontrast group and 50\% core infarct overestimation in the contrast 


\begin{tabular}{lcccc}
\hline \multicolumn{1}{c}{ Characteristic } & $\begin{array}{c}\text { Recent IV } \\
\text { Contrast }^{\mathbf{a}}\end{array}$ & $\begin{array}{c}\text { No Recent IV } \\
\text { Contrast }^{\mathbf{a}}\end{array}$ & $\begin{array}{c}\text { Overall }^{\mathrm{a}} \\
\text { Oalue }^{\mathrm{b}}\end{array}$ \\
\hline Count & 23 & 15 & 38 & $\mathrm{NA}$ \\
Age $(\mathrm{yr})$ & $70.5 \pm 16.0$ & $76.5 \pm 18.4$ & $72.9 \pm 17.0$ & .32 \\
Serum creatinine level (mg/dL) & $1.08 \pm 0.34$ & $1.12 \pm 0.29$ & $1.10 \pm 0.32$ & .78 \\
Female & $15(65 \%)$ & $7(47 \%)$ & $22(58 \%)$ & .32 \\
Time since last known well (hr) & $12.5 \pm 5.6$ & $9.3 \pm 7.8$ & $11.2 \pm 6.6$ & .19 \\
ASPECTS & $5.4 \pm 2.0$ & $7.5 \pm 2.2$ & $6.2 \pm 2.3$ & .005 \\
CTP core (median) (range) (mL) & $4(0-234)$ & $13(0-208)$ & $6(0-234)$ & .28 \\
Collateral grade & & & & .71 \\
1 & $15(65 \%)$ & $9(60 \%)$ & $24(63 \%)$ & \\
2 & $6(26 \%)$ & $3(20 \%)$ & $9(24 \%)$ & \\
3 & $2(9 \%)$ & $3(20 \%)$ & $5(13 \%)$ & \\
Thrombectomy & $11(48 \%)$ & $12(80 \%)$ & $23(61 \%)$ & .09 \\
\hline
\end{tabular}

Note:-NA indicates not applicable.

${ }^{a}$ Values are No., No. (\%), or mean \pm SD.

${ }^{\mathrm{b}} P$ values are from the Fisher exact test or 2 -sample $t$ test with unequal variances.

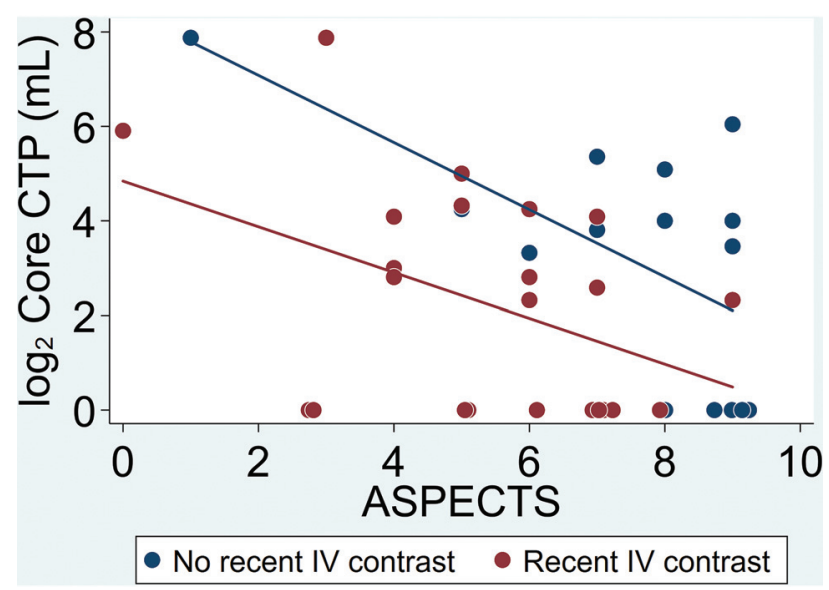

FIG 1. Scatterplot of CT-ASPECTS versus log-transformed CTP-estimated core infarct for the recent IV contrast group (red) and the contrast-naïve group (blue) with lines of best fit. For the same CTASPECTS, the CTP-estimated core infarct is consistently less in the recent IV contrast group with divergence of the lines of best fit toward the lower ASPECTS indicative of more substantial differences between the 2 cohorts and more significant underestimation of estimated core infarct for lower CT-ASPECTS.

group based on prior experience, resulting in 14 patients in each cohort with an $\alpha$ level of .05, a $\beta$ level of 0.2 , and power of $80 \%$. The Fischer exact test and unequal variances 2 -sample $t$ test were used for categoric and continuous variables, respectively, to assess differences between the groups.

A multivariate linear regression model was produced to test whether RAPID-estimated core infarct was associated with recent IV contrast administration, while including variables that met a predetermined statistical significance threshold $(P=.05)$. Core infarct estimated by RAPID values underwent log-transformation before analysis to accommodate overly influential outliers and allow improved adherence to the assumption of the linear regression model of homoscedasticity and normally distributed residuals. An absolute value of 1 was added to the core infarct estimated value before log-transformation because a log-transformation of zero is not possible.

Regression results are presented as exponentiated regression coefficients labeled as proportional increases (PIs). For example, a

\section{RESULTS}

PI of 1.2 would indicate a $20 \%$ increase, whereas a PI of 0.8 would indicate a $20 \%$ decrease. Data analysis was conducted on STATA 15.1 (StataCorp [2017], STATA Statistical Software, Release 15).

In an effort to determine whether RAPID was underestimating the presentation core infarct value, we used multivariable regression analysis to determine whether there was a difference in final infarct volumes compared with that estimated by RAPID in patients who were contrast-naïve and those who received prior contrast, while adjusting for the time from CTP to reperfusion. For this particular analysis, core infarct values were not log-transformed.

Of the 38 patients who met the inclusion criteria, 23 received recent IV contrast and 15 were contrast-naïve. Table 1 summarizes group and overall characteristics.

The average age was 72.9 years, and $58 \%$ were female, with no significant difference between groups for either. There was no difference in serum creatinine levels, a marker of renal function, between those who received recent IV contrast and the contrastnaïve group $(1.08 \pm 0.34$ versus $1.12 \pm 0.29, P=.78)$. The recent IV contrast group tended to have longer times since LKW at the time of CTP imaging but not significantly so (12.5 versus 9.3 hours, $P=.19$ ). While those who received recent IV contrast demonstrated significantly lower ASPECTS (5.4 versus 7.5, $P=.005$ ), there was not a statistically significant baseline difference in core infarct estimates by RAPID between the 2 cohorts $(P=.28)$ (Fig 1$)$. There was no significant difference in CTA collateral grades $(P=.71)$. Contrast-naïve patients were more likely to be selected for MT but not significantly so ( $80 \%$ versus $48 \%, P=.09$ ).

Final multivariable linear regression results are shown in Table 2, with the log-transformed core infarct estimated by RAPID as the outcome, and included the following predictors: 1) recent IV contrast administration, 2) ASPECTS, and 3) collateral grade. Recent IV contrast administration was associated with decreased (about two-thirds) RAPID-estimated core infarct (PI $=0.34 ; 95 \%$ CI, 0.12-0.96; $P=.04$ ). Most important, analysis of the data before logarithmic transformation demonstrated an even more significant decrease in RAPID-estimated core infarct with recent IV contrast administration. There was an inverse relationship between the ASPECTS and core infarct estimated by RAPID, with each point increase in the ASPECTS resulting in 26\% lower core infarct estimated by RAPID (PI $=0.74 ; 95 \% \mathrm{CI}, 0.59-0.93 ; P=.01$ ). Patients with intermediate and poor collaterals (grades 2 and 3, respectively) demonstrated higher core infarcts by RAPID than patients with excellent collaterals $(\mathrm{PI}=2.27 ; 95 \% \mathrm{CI}, 0.78-6.60 ; P=.13$ and $\mathrm{PI}=8.46 ; 95 \% \mathrm{CI}, 2.10-34.06 ; P=.004$, respectively). There were no associations between RAPID-estimated core infarct with other variables, including age, sex, and time since LKW, and these variables were therefore excluded. 
Seventeen patients met the inclusion criteria for analysis comparing final infarct volume with the core infarct estimated by RAPID ( 8 received prior contrast and 9 were contrast-naïve) (Fig 2). The average times from CTP to reperfusion were not significantly different

Table 2: Multivariable linear regression results

\begin{tabular}{lccc}
\hline \multicolumn{1}{c}{ Characteristic } & $\mathbf{P I}^{\mathbf{a}}$ & $\begin{array}{c}\text { 95\% Confidence } \\
\text { Interval }\end{array}$ & $\begin{array}{c}\boldsymbol{P} \\
\text { Value }\end{array}$ \\
\hline Recent IV contrast & 0.34 & $(0.12-0.96)$ & .04 \\
ASPECTS & 0.74 & $(0.59-0.93)$ & .01 \\
$\begin{array}{l}\text { Collateral grade }(1= \\
\text { reference group) }\end{array}$ & & & \\
2 & & & \\
3 & 2.27 & $(0.78-6.60)$ & .13 \\
\hline
\end{tabular}

${ }^{\text {a }}$ Outcome is $\log 2$ core CTP.

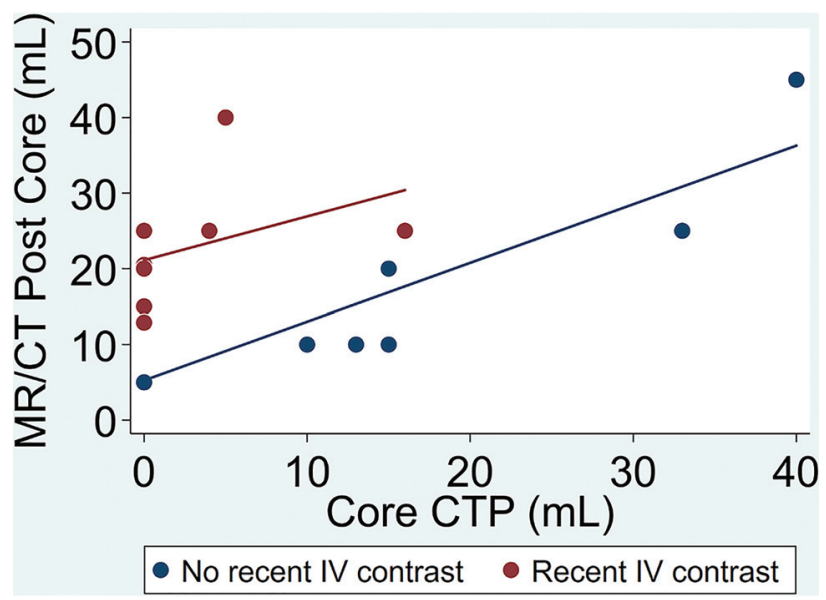

FIG 2. Scatterplot of CTP-measured core infarct volume versus postoperative core infarct volume as measured by MR imaging or CT for the recent IV contrast group (red) and the contrast-naïve group (blue) with lines of best fit. For the same preoperative CTP-measured core, the postoperative core infarct is greater in the recent IV contrast group, suggesting that recent IV contrast underestimates CTP-measured core infarct volume at presentation.
$(P=.37)$. While the slopes of the regression lines were similar $(P=.61)$, final infarct volume was higher for any given core infarct volume estimated by RAPID in patients who received prior contrast (coefficient $=14.4 ; 95 \% \mathrm{CI}, 5.7-23.0 ; P=.003$ ). In other words, in patients who received contrast before CTP at our institution, RAPID underestimated the infarct volume.

\section{DISCUSSION}

In early 2018, synchronous with rapidly increasing CTP imaging using RAPID postprocessing, our team observed a repeat phenomenon: Extended window ELVOs with conspicuous MCA territory hypoattenuation on NCCT (Fig 3) were frequently demonstrating disproportionately small core infarcts as predicted by RAPID (Fig 4), in contradistinction to patients presenting directly to our institution (Fig 5) who underwent CTP (Fig 6). This seemingly occurred exclusively in our acute ischemic stroke transfers, essentially all of whom received IV iodinated contrast for CTA to confirm ELVO before transfer.

The results of this study corroborate our hypothesis: RAPID postprocessing tends to underestimate core infarct volume (defined as $\mathrm{rCBF}$ of $<30 \%$ ) in patients receiving IV contrast for a separate imaging study within 8 hours of CTP imaging. While multifarious pitfalls of RAPID software have been described, particularly overestimation of core infarct in the hyperacute setting, this is, to our knowledge, the first description of underestimation of core infarct related to recent IV contrast administration. ${ }^{8-10}$

Our findings have impacted our team's algorithm for transfer patients with ELVO by decreasing our reliance on RAPID output. This may additionally impact other stroke centers as we continue learning which patients will benefit from this life-saving procedure.

In this era of perpetually increasing extended window MT volumes, clinicians must be cognizant of this pitfall because estimation of core infarct is clinically relevant. First, in patients with extended windows eligible for MT by the DAWN criteria, the baseline ASPECTS appears to modify the treatment effect of MT, with significantly greater benefit in patients with higher ASPECTS of 7-10. ${ }^{11}$ Second, MT in the setting of an ASPECTS of $0-5$ is often more complex, is associated with worse clinical outcomes, and has an increased symptomatic intracranial hemorrhage risk. $^{5}$ While some neurointerventionalists advocate MT even in the presence of ASPECTS of 3-5, awareness of this phenomenon may augment the accuracy of patient prognostication, leading to a more informed decision to intervene, family discussion, and procedural consent.

RAPID-generated mismatch maps comprise the following: time-tomaximum of $>6.0$ seconds, indicative of ischemic penumbra likely to infarct without reperfusion; and $\mathrm{rCBF}$ of $<30 \%$, a surrogate for irreversibly injured parenchyma. RAPID software, however, does not directly identify infarcted tissue; rather, the perfusion
FIG 3. A 56-year-old man who presented to an outside hospital with a right MCA syndrome and ELVO confirmed on CTA. Last known well was approximately 11 hours before imaging at our institution. A, NCCT demonstrates loss of gray-white matter differentiation, in keeping with acute infarct, involving the right insula as well as the frontal and temporal opercula. B, CTA confirms a right M1 segment (yellow circle) occlusion. C, DWI reveals an extensive area of acute infarct correlating with the areas of hypoattenuation on the NCCT. TICI 3 reperfusion was achieved within 60 minutes of the CTP study, and the MR imaging was obtained later in the day. 


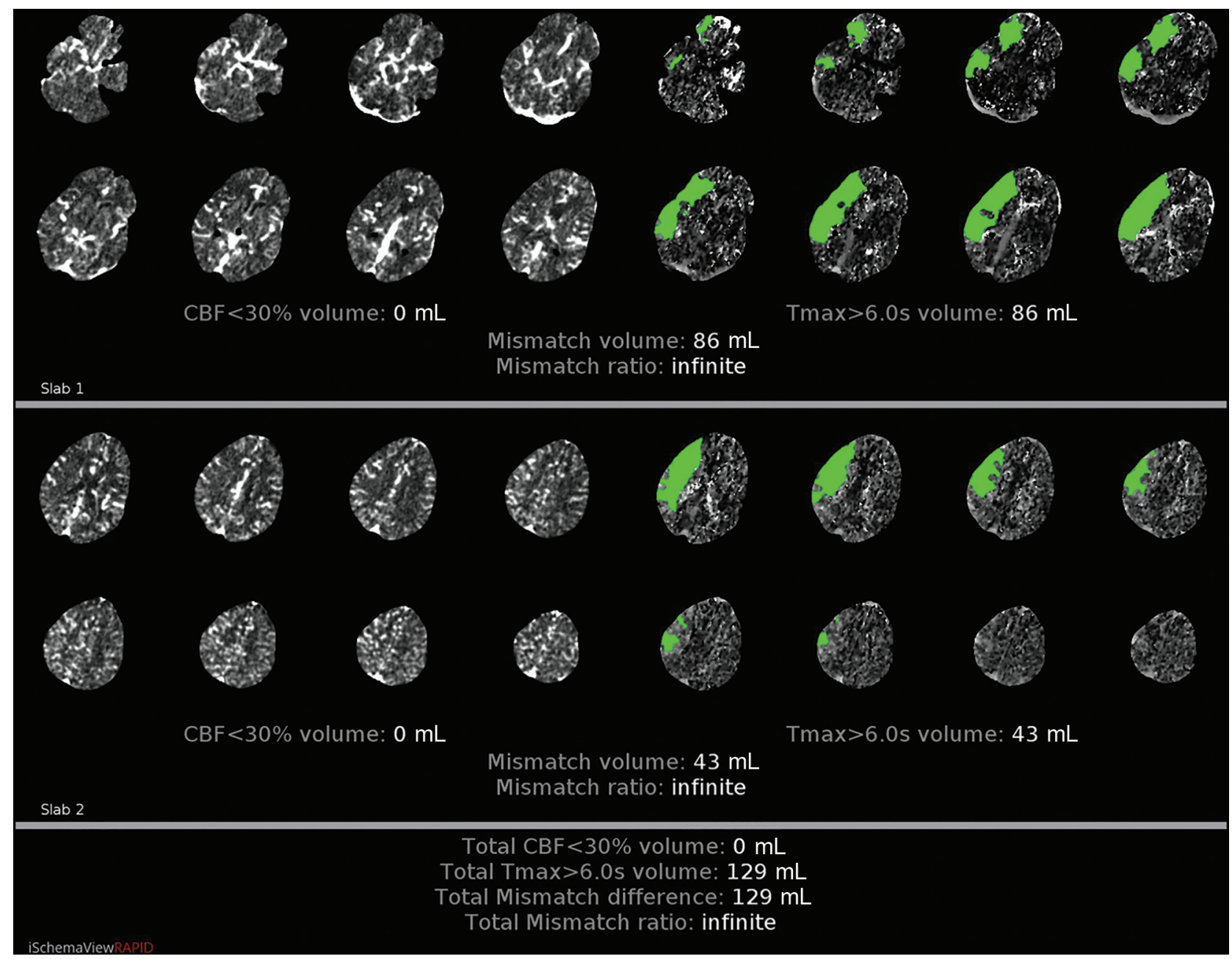

FIG 4. CTP with RAPID postprocessing from the same patient as in Fig 3 suggests no core infarct (lack of pink color-coding and an rCBF of $<30 \%$ volume of $0 \mathrm{~mL}$ ) with a large area of ischemic penumbra (green color-coding with time-to-maximum [Tmax] of $>6 \mathrm{~seconds}$ of $129 \mathrm{~mL}$ ).

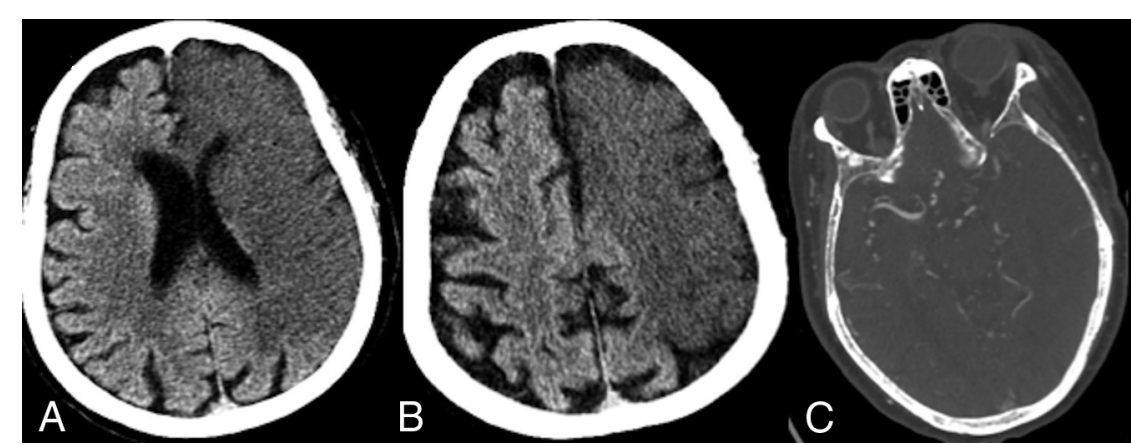

FIG 5. A 67-year-old man who presented directly to our institution (no recent IV contrast) with a left MCA syndrome and last known well approximately 8 hours before imaging. $A$ and $B$, NCCT demonstrates hypoattenuation involving a large volume of the left MCA and anterior cerebral artery territories. C, CTA demonstrates a internal carotid terminus occlusion with involvement of the carotid terminus and $\mathrm{Al}$ and $\mathrm{Ml}$ segments.

maps reflect cerebral hemodynamics at the moment the scan was performed. Spontaneous or posttherapeutic recanalization to a recently infarcted territory may result in failure of the RAPID software to detect core infarct using the $\mathrm{rCBF}$ of $<30 \%$ threshold; however, all our included patients had confirmed ELVO at the time of imaging at our institution (Figs 3 and 5). Alternatively, recruitment of leptomeningeal collaterals to acutely infarcted parenchyma may result in a false-negative core on RAPID due to similar principles affecting CTP parameters. The automated suppression of markedly hypoattenuating regions in RAPID, such as chronic infarcts or CSF-containing structures, may lead to suppression of acutely infarcted tissue, particularly in the late extended window with more of a subacute infarct CT appearance. These pitfalls, however, cannot explain the striking discrepancy between our cohorts.

The ideal rCBF threshold should err on the side of underestimating core infarct to avoid inappropriate exclusion of potential MT candidates. Accordingly, rCBF of $<30 \%$ underestimates infarcts present on DWI by an average of $12 \mathrm{~mL}$ but demonstrates 


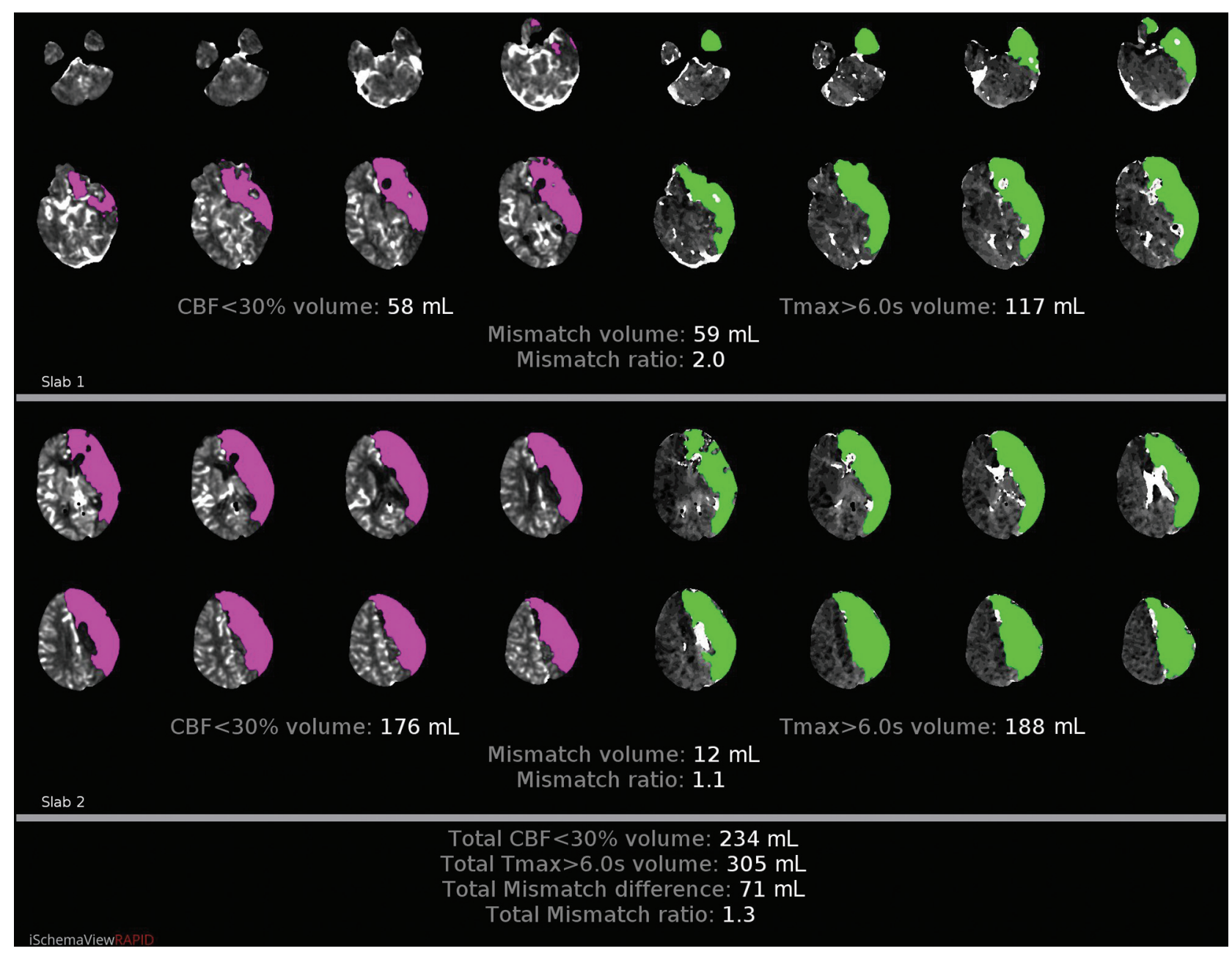

FIG 6. CTP imaging with RAPID postprocessing from the same patient as in Fig 5 suggests an extensive area of core infarct (pink color-coding and an $\mathrm{rCBF}$ of $<30 \%$ volume of $234 \mathrm{~mL}$ ) corresponding to the large volume of hypoattenuation on NCCT. Tmax indicates time-to-maximum.

greater specificity in predicting DWI-positive voxels compared with an $\mathrm{rCBF}$ of $<38 \%$, which is actually more accurate, on average, in estimating core infarct. ${ }^{7}$ No perfusion parameter should be assessed in isolation. $\mathrm{rCBF}$ should be analyzed in conjunction with the NCCT, CTA, as well as the additionally provided perfusion parameters, particularly $\mathrm{CBV}$. $\mathrm{CBV}$ of $<40 \%$ of the contralateral hemisphere, absolute CBV of $<2 \mathrm{~mL} / 100 \mathrm{~g}^{-1}$, and relative CBV of 0.32 have all been shown to be predictive of core infarct. ${ }^{12-14}$ Multiple studies, however, have demonstrated rCBF to be the optimal parameter for estimating irreversible infarction, including studies using concurrent DWI performing significantly better than $\mathrm{CBV}{ }^{7,15,16}$ More important, $\mathrm{rCBF}$ of $<30 \%$ is the surrogate for core infarct used in multiple contemporary thrombectomy trials and is endorsed by the major endovascular societies. ${ }^{2,3,17-20}$ We therefore used $\mathrm{rCBF}$ of $<30 \%$ for our analysis; however, we routinely assess NCCT for ASPECTS, CTA for collaterals, as well as additional perfusion parameters, depending on specific clinical scenarios.

While the recent IV contrast group presented with significantly lower CT ASPECTS, likely due to a longer time from LKW to CTP, there was no significant difference in RAPID core infarct estimates. When we controlled for ASPECTS and other associated variables, recent IV contrast administration was associated with a two-thirds decrease in the RAPID-predicted core infarct volume for the same ASPECTS. This may result in inappropriately selecting patients for MT on the basis of the DAWN or DEFUSE-3 inclusion criteria. ${ }^{2,3}$

Multitudinous factors contribute to CTP variability, including timing and volume of IV contrast administration, postprocessing algorithm (eg, definition of arterial input/venous output function, motion correction, and smoothing), and parameter threshold. Although CTP use is increasing, consensus has not been achieved among manufacturers as to which parameter optimally defines core infarct. Significant variability in calculated core infarct volumes has been demonstrated among various software packages. ${ }^{21}$

In our limited series, postprocessing of the CTP data using the AW Server software package (GE Healthcare; https://www. gehealthcare.com/products/advanced-visualization/platforms/ aw-server) without a smoothing algorithm resulted in more conspicuous reduction of $\mathrm{rCBF}$ in the ischemic core than the RAPID software package maps, which do use a smoothing algorithm (Fig 7).

The precise mechanism of recent IV contrast resulting in falsely low core infarcts by RAPID remains a conundrum. The sequence of CTA and CTP in stroke CT protocols has been shown to have no significant influence on quantitative parameters of CTP. ${ }^{22}$ 


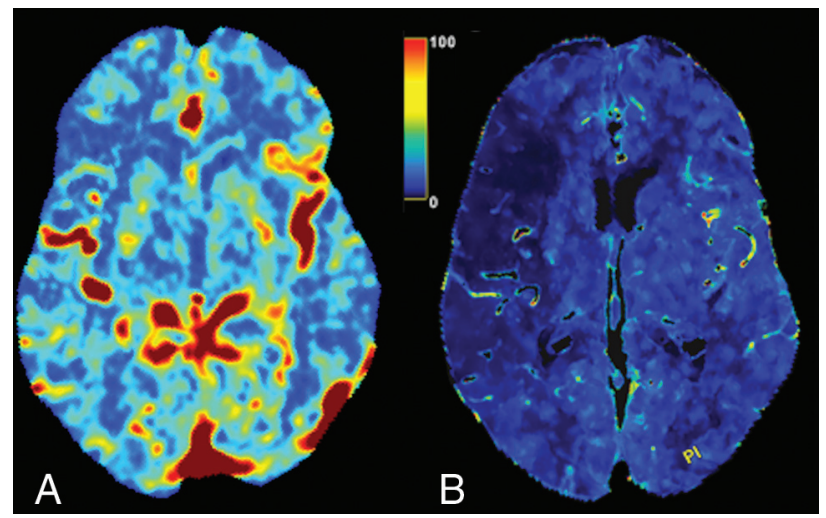

FIG 7. The same 56-year-old man with right $\mathrm{M} 1$ segment occlusion as presented in Fig 3. A, rCBF map from the RAPID postprocessing of CTP data demonstrates subtle decreased blood flow to the right MCA territory corresponding to the suggested core infarct volume of $0 \mathrm{~mL}$. $B, \mathrm{rCBF}$ map from GE Healthcare processing of the CTP data demonstrates much more conspicuous decreased blood flow within the right MCA territory (scale bar $=0-100 \mathrm{~mL} / \mathrm{g} / \mathrm{min}$ ).

However, IV contrast administered several hours before CTP, as opposed to several minutes, may allow sufficient time for the contrast to circulate to alter perfusion parameters. Our group and others have previously described contrast staining of acutely infarcted brain parenchyma on NCCT following digital subtraction angiography. ${ }^{23-26}$ Similarly, contrast administered several hours before CTP may lead to progressive accumulation in the infarcted tissue, thereby elevating the Hounsfield units. The underlying mechanism for such contrast staining is likely disruption of the blood-brain barrier. $^{27}$

The authors are cognizant of ASPECTS shortcomings and readily acknowledge that it is not the criterion standard for estimating core infarction. CT lacks the sensitivity and specificity of MR imaging in the detection of acute infarction. ${ }^{28,29}$ Nevertheless, the ASPECTS remains pertinent because it allows expeditious assessment and prognostication before treatment. Several of the major ELVO thrombectomy trials excluded ASPECTS of $<6$ from $\mathrm{MT}$, and the ACR-ASNR-SIR-SNIS practice parameter ${ }^{20}$ supports the same exclusion criterion. ${ }^{17-19}$ Although individual ASPECTS regions are weighted unequally in regard to volume, several studies have shown correlation between ASPECTS and CTP-estimated ischemic core. ${ }^{11,30}$ While CTP measures collateral perfusion and does not directly measure tissue viability, ASPECTS scoring is based on hypoattenuation, which is highly specific for irreversible parenchymal injury and becomes more apparent in the extended window. ${ }^{31,32}$ Nevertheless, our final infarct volume subanalysis in patients who had a good result from thrombectomy also supports the hypothesis. While the slopes of the regression lines for each cohort were similar, the y-intercept was significantly higher in patients who received prior contrast while controlling for the time between CTP acquisition and time to reperfusion. In other words, RAPID was underestimating the final infarct volume in patients who had received prior contrast.

This study has limitations inherent in its retrospective nature and relatively small sample size. Many patients who underwent CT stroke protocol imaging were ultimately diagnosed with distal branch occlusions, lacunar infarcts, intracranial hemorrhage, as well as nonvascular conditions and were therefore excluded. In addition, patients with ASPECTS of 10 were excluded. Heterogeneity in follow-up imaging at our institution, largely at the discretion of the neurology team, also limited our sample sizes for final infarct analysis. Given the small sample size, we could not analyze matched ASPECTS strata between the 2 cohorts. The small sample size is largely due to the narrow time window (May 2018 to January 2019) for inclusion in this study. Rather than delay the reporting of such a clinically relevant pitfall to augment our sample size, we thought it pertinent to alert our neuroradiology and neurointerventional colleagues to our findings.

Another limitation is our lack of a criterion standard reference for core infarct estimation. This is due to the nature of the study because no patients underwent MR imaging simultaneous with the baseline NCCT. Patients excluded from thrombectomy would likely have a more significant interval growth of core infarct on follow-up compared with those who were successfully recanalized; therefore, a direct comparison on follow-up imaging would be misleading. Regardless, our results are striking irrespective of the sample, and it is, therefore, critical that all centers relying on RAPID as part of their selection criteria for MT be cognizant of this phenomenon.

\section{CONCLUSIONS}

Recent administration of IV iodinated contrast, omnipresent among stroke transfers undergoing CTA to confirm ELVO before transfer, may result in falsely low core infarcts as predicted by RAPID software. It is critical for neurointerventionalists and clinicians to peruse the NCCT for evidence of hypoattenuated parenchyma and to estimate a baseline ASPECTS, particularly in the extended window with recent administration of IV iodinated contrast.

Disclosures: Steven W. Hetts_UNRELATED: Consultancy: Route 92 Medical, Comments: clinical trial clinical event evaluator for thrombectomy device company; Grants/Grants Pending: Siemens, Stryker, MicroVention, Terumo, Comments: research contract for advanced angiography suites, core imaging lab for aneurysm treatment clinical trials*; Stock/Stock Options: ThrombX, Comments: investor in startup thrombectomy device company. Jeffrey Nelson—RELATED: Grant: National Institutes of Health.* Matthew R. Amans-RELATED: Grant: National Institutes of Health, Comments: Research reported in this publication was supported by the National Institute of Deafness and Other Communication Disorders R21DC01608701A1 and the National Heart, Lung, and Blood Institute R56HL149124-01*; UNRELATED: Consultancy: Covidien, Stryker, MicroVention; Expert Testimony: various medicolegal consulting. *Money paid to the institution.

\section{REFERENCES}

1. Cagnazzo F, Derraz I, Dargazanli C, et al. Mechanical thrombectomy in patients with acute ischemic stroke and ASPECTS $\leq 6$ : a metaanalysis. J Neurointerv Surg 2020;12:350-55 CrossRef Medline

2. Nogueira RG, Jadhav AP, Haussen DC, et al; DAWN Trial Investigators. Thrombectomy 6 to 24 hours after stroke with a mismatch between deficit and infarct. N Engl J Med 2018;378:1121 CrossRef Medline

3. Albers GW, Lansberg MG, Kemp S, et al. A multicenter randomized controlled trial of endovascular therapy following imaging evaluation for ischemic stroke (DEFUSE 3). Int J Stroke 2017;12:896-905 CrossRef Medline

4. Yoo AJ, Zaidat OO, Chaudhry ZA, et al; Penumbra Pivotal and Penumbra Imaging Collaborative Study (PICS) Investigators. Impact of pretreatment noncontrast CT Alberta Stroke Program Early CT Score on clinical outcome after intra-arterial stroke therapy. Stroke 2014;45:746-51 CrossRef Medline 
5. Schröder J, Thomalla G. A critical review of Alberta Stroke Program Early CT Score for evaluation of acute stroke imaging. Front Neurol 2016;7:245 CrossRef Medline

6. Nambiar V, Sohn SI, Almekhlafi MA, et al. CTA collateral status and response to recanalization in patients with acute ischemic stroke. AJNR Am J Neuroradiol 2014;35:884-90 CrossRef Medline

7. Cereda CW, Christensen S, Campbell BC, et al. A benchmarking tool to evaluate computer tomography perfusion infarct core predictions against a DWI standard. J Cereb Blood Flow Metab 2016;36:1780-89 CrossRef Medline

8. Boned S, Padroni M, Rubiera M, et al. Admission CT perfusion may overestimate initial infarct core: the ghost infarct core concept. $J$ Neurointerv Surg 2017;9:66-69 CrossRef Medline

9. Angermaier A, Khaw AV, Kirsch M, et al. Influence of recanalization and time of cerebral ischemia on tissue outcome after endovascular stroke treatment on computed tomography perfusion. $J$ Stroke Cerebrovasc Dis 2015;24:2306-12 CrossRef Medline

10. Silvennoinen HM, Hamberg LM, Lindsberg PJ, et al. CT perfusion identifies increased salvage of tissue in patients receiving intravenous recombinant tissue plasminogen activator within 3 hours of stroke onset. AJNR Am J Neuroradiol 2008;29:1118-23 CrossRef Medline

11. Bhuva P, Yoo AJ, Jadhav AP, et al; DAWN Trial Investigators. Noncontrast computed tomography Alberta Stroke Program Early CT Score may modify intra-arterial treatment effect in DAWN. Stroke 2019;50:2404-12 CrossRef Medline

12. Srinivasan A, Goyal M, Al Azri F, et al. State-of-the-art imaging of acute stroke. Radiographics 2006;26(Suppl 1):S75-95 CrossRef Medline

13. Wintermark M, Flanders AE, Velthuis B, et al. Perfusion-CT assessment of infarct core and penumbra: receiver operating characteristic curve analysis in $\mathbf{1 3 0}$ patients suspected of acute hemispheric stroke. Stroke 2006;37:979-85 CrossRef Medline

14. Mokin M, Levy EI, Saver JL, et al; SWIFT PRIME Investigators. Predictive value of RAPID assessed perfusion thresholds on final infarct volume in SWIFT PRIME (Solitaire With the Intention for Thrombectomy as Primary Endovascular Treatment). Stroke 2017;48:932-38 CrossRef Medline

15. Amukotuwa S, Straka M, Aksoy D, et al. Cerebral blood flow predicts the infarct core: new insights from contemporaneous diffusion and perfusion imaging. Stroke 2019;50:2783-89 CrossRef Medline

16. Campbell BC, Christensen S, Levi CR, et al. Cerebral blood flow is the optimal CT perfusion parameter for assessing infarct core. Stroke 2011;42:3435-40 CrossRef Medline

17. Demchuk AM, Goyal M, Menon BK, et al; ESCAPE Trial Investigators. Endovascular Treatment for Small Core and Anterior Circulation Proximal Occlusion with Emphasis on Minimizing CT to Recanalization Times (ESCAPE) trial: methodology. Int J Stroke 2015;10:429-38 CrossRef Medline

18. Saver JL, Goyal M, Bonafe A, et al; SWIFT PRIME Investigators. Solitaire $^{\mathrm{TM}}$ with the Intention for Thrombectomy as Primary Endovascular Treatment for Acute Ischemic Stroke (SWIFT PRIME) trial: protocol for a randomized, controlled, multicenter study comparing the Solitaire revascularization device with IV tPA with IV IPA alone in acute ischemic stroke. Int J Stroke 2015;10:439-48 CrossRef Medline
19. Molina CA, Chamorro A, Rovira À, et al. REVASCAT: a randomized trial of revascularization with SOLITAIRE FR device vs best medical therapy in the treatment of acute stroke due to anterior circulation large vessel occlusion presenting within eight-hours of symptom onset. Int J Stroke 2015;10:619-26 CrossRef Medline

20. ACR-ASNR-SIR-SNIS Practice Parameter for the Performance of Endovascular Embolectomy and Revascularization in Acute Stroke. 2018. https://www.acr.org/-/media/ACR/Files/Practice-Parameters/ Acute-Stroke.pdf?la=en. Accessed February 25, 2020

21. Koopman MS, Berkhemer OA, Geuskens RR, et al; MR CLEAN Trial Investigators. Comparison of three commonly used CT perfusion software packages in patients with acute ischemic stroke. J Neurointerven Surg 2019;11:1249-56 CrossRef Medline

22. Dorn F, Liebig T, Muenzel D, et al. Order of CT stroke protocol (CTA before or after CTP): impact on image quality. Neuroradiology 2012;54:105-12 CrossRef Medline

23. Amans MR, Cooke DL, Vella M, et al. Contrast staining on CT after DSA in ischemic stroke patients progresses to infarction and rarely hemorrhages. Interv Neuroradiol 2014;20:106-15 CrossRef Medline

24. Nakano S, Iseda T, Yoneyama T, et al. Early CT signs in patients with acute middle cerebral artery occlusion: incidence of contrast staining and haemorrhagic transformations after intra-arterial reperfusion therapy. Clin Radiol 2006;61:156-62 CrossRef Medline

25. Nakano S, Iseda T, Kawano H, et al. Parenchymal hyperdensity on computed tomography after intra-arterial reperfusion therapy for acute middle cerebral artery occlusion: incidence and clinical significance. Stroke 2001;32:2042-48 CrossRef Medline

26. Parrilla G, García-Villalba B, Espinosa de Rueda M, et al. Hemorrhage/ contrast staining areas after mechanical intra-arterial thrombectomy in acute ischemic stroke: imaging findings and clinical significance. AJNR Am J Neuroradiol 2012;33:1791-96 CrossRef Medline

27. Numaguchi Y, Fleming MS, Hasuo K, et al. Blood-brain barrier disruption due to cerebral arteriography: CT findings. J Comput Assist Tomogr 1984;8:936-39 CrossRef Medline

28. Jaillard A, Hommel M, Baird AE, et al. Significance of early CT signs in acute stroke: a CT scan-diffusion MRI study. Cerebrovasc Dis 2002;13:47-56 CrossRef Medline

29. Kim EY, Ryoo JW, Roh HG, et al. Reversed discrepancy between CT and diffusion-weighted MR imaging in acute ischemic stroke. AJNR Am J Neuroradiol 2006;27:1990-95 Medline

30. Haussen DC, Dehkharghani S, Rangaraju S, et al. Automated CT perfusion ischemic core volume and noncontrast CT ASPECTS (Alberta Stroke Program Early CT Score): correlation and clinical outcome prediction in large vessel stroke. Stroke 2016;47:2318-22 CrossRef Medline

31. Lev MH, Farkas J, Gemmete JJ, et al. Acute stroke: improved nonenhanced CT detection-benefits of soft-copy interpretation by using variable window width and center level settings. Radiology 1999;213:150-55 CrossRef Medline

32. Dzialowski I, Weber J, Doerfler A, et al. Brain tissue water uptake after middle cerebral artery occlusion assessed with CT. J Neuroimaging 2004;14:42-48 CrossRef Medline 